\title{
New Insights Into The Narrow-Line Seyfert 1 Phenomenon
}

\author{
Smita Mathur \\ Department of Astronomy, 140 West 18th Avenue, The Ohio State University, \\ Columbus, Ohio 43210, USA
}

\begin{abstract}
I briefly review the X/UV absorber models and show that the observations of NLS1s are generally consistent with the models. The covering factor of absorbers in NLS1s is likely to be high and there is some evidence of super-solar metallicities. I argue that NLS1s may be active galaxies in the early stage of their evolution and as such, may be low luminosity, low redshift analogues of the high redshift quasars. NLS1s may reside in rejuvenated gas-rich galaxies. I also propose that the high Fe II emission in NLS1s may be a direct consequence of their large accretion rate and so a collisional ionization origin of Fe II is favored.
\end{abstract}

Key words: galaxies: active; quasars: general; quasars: absorption lines; X-rays: galaxies

\section{Introduction}

The organizers of this workshop have asked me to talk about the X-ray/UV absorbers in AGN and discuss what they can tell us about the Narrow Line Seyfert 1 phenomenon. However, I am going to go well beyond my expertise and speculate. I think that this meeting is an ideal platform for discussing new ideas, so let me start with my conclusions first and get them out of the way. Conclusions:

(1) The covering factor of warm absorbers is likely to be high in NLS1s.

(2) Metallicities might be super solar.

(3) NLS1s may be AGN in the making and as such, may be low redshift, low luminosity analogues of the high redshift quasars.

(4) NLS1s reside in rejuvenated/ gas rich galaxies.

Having said that, let's start from the beginning. 


\subsection{The $X / U V$ Absorbers}

The UV and X-ray intrinsic absorption systems observed in active galactic nuclei (AGN) were never thought to be related to one another because of their apparently different physical properties. The inferred total column density of UV absorption lines was believed to be about $10^{20} \mathrm{~cm}^{-2}$, and the ionization parameter U was thought to be such that CIV would be the dominant ionization phase of carbon. In contrast, the cold X-ray absorbers are neutral with column densities $>10^{21} \mathrm{~cm}^{-2}$. Models of warm X-ray absorbers also required larger column densities and ionization parameters than the UV absorbers. As was shown later, the ionization structure of the absorbers was what was lacking from our understanding.

The above situation changed with the quasi-simultaneous observations of 3C351 with the ROSAT PSPC and the HST FOS. The UV spectrum of 3C351 showed OVI absorption line doublets and the X-ray spectrum showed edges due to OVII/OVIII. These observations lead Mathur et al. (1994) to conclude that the UV and X-ray absorbers are in fact one and the same. The combined UV and X-ray analysis proved to be a powerful technique to probe the physical properties of the nuclear regions of AGN: high column density, highly ionized, outflowing material situated outside the broad emission line region. In addition, the density of the absorber could be constrained in variable systems. This was a previously unknown component with important consequences: an outflow that carries a significant amount of kinetic energy, and with a mass outflow rate comparable to the accretion rate needed to power the AGN engine.

Later work supported the unified X/UV absorber scenario (Mathur 1994, Mathur et al. 1995, 1997, 1998, 1999, Shields \& Hamann 1997a, Crenshaw 1997). I will defer to Mathur (1997) for a discussion of controversies regarding the X/UV models. I conclude that all the observed data support this picture: the UV and X-ray absorbers are physically related to each other. At the very least, the X-ray absorbers make a substantial contribution to the absorption seen in the UV.

\section{X-ray \& UV Absorption in NLS1s}

Table 1 lists some NLS1 galaxies showing absorption in X-rays and/or UV. I have compiled this list from the literature and it is by no means complete. There are a few more ROSAT selected NLS1s with X-ray warm absorbers for which UV spectra are not available. 
Table 1

NLS1s with X-ray and/or UV Absorbers

\begin{tabular}{|lccc|}
\hline \hline Object & $\begin{array}{c}\text { X-ray Warm } \\
\text { Absorber }\end{array}$ & UV & Comments \\
& Absorber & \\
\hline IRAS 13349+2438 & Y & Y (HST) & Only CIV, low S/N \\
Mrk 1298 & Y & Y (IUE) & Low S/N in IUE \\
I Zw 1 & Y & Y (HST) & Excess in blue wing \\
& & & of UV emission lines. \\
NGC4051 & Y & N? & -do- \\
Mrk 507 & Y & $?$ & Not expected \\
IRAS 17020+4544 & Y & $?$ & (too ionized) \\
Q0117-2837 & Y & N (IUE) & -do- \\
RX J 0134.3-4258 & Y & $?$ & \\
PG 1404+266 & Y/N & Y & \\
\hline
\end{tabular}

From Table 1 we see that for some NLS1s with X-ray warm absorbers either (1) UV data do not exist, or (2) UV absorption is not expected because the $\mathrm{X}$-ray absorber is very highly ionized. I cannot comment on the X/UV connection for these objects. Excluding these objects, we have warm absorbers with either (1) UV absorption, or (2) blue asymmetric emission lines. The first case is what is expected from the X/UV absorber models, while the second (blue asymmetric emission lines) is a new observation and is intriguing. I would like to propose that the blue asymmetry is caused by emission from the outflowing/inflowing warm absorber. (See also B. Peterson's article on the broad HeII emission line in NGC4051). A preliminary investigation shows consistency with the predictions from photoionization models if the covering factor is large. This leads to my first conclusion: Warm absorbers in NLS1s have large covering factors.

Now consider the warm absorbers with UV absorbers. The existence of UV absorbers is in itself favored by the unified X/UV models. There are three such objects listed in Table 1: (1) IRAS 13349+2438: the ROSAT spectrum of this object is discussed in Brandt, Mathur, Reynolds \& Elvis (1997). Using photoionization models and the parameters of warm absorbers, we can predict the column density of UV absorption lines. HST FOS observed the CIV absorption line in this object (Figure 1). Being a single orbit snapshot observation, the signal-to-noise ratio is poor. Also, with just one line, the result cannot be 


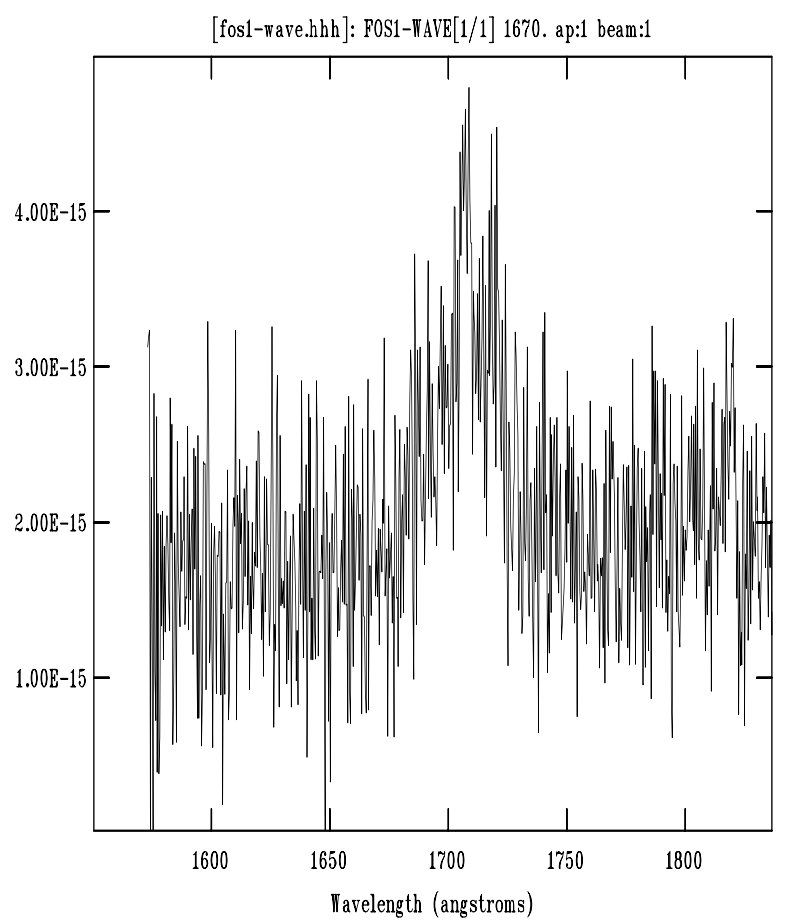

Fig. 1. One orbit HST FOS spectrum of IRAS $13349+2438$ showing associated CIV absorption. Wavelength in angstroms is plotted on the $\mathrm{X}$-axis, while Y-axis corresponds to flux in units of ergs $\mathrm{s}^{-1} \mathrm{~cm}^{-2} \AA^{-1}$.

conclusive. Nevertheless, the observed CIV column density is as predicted for a reasonable value of the $\mathrm{b}$ parameter, $\mathrm{b} \sim 100 \mathrm{~km} / \mathrm{s}$. (2) I Zw 1: This object has a weak warm absorber, and as expected, a weak associated absorption line system of Ly $\alpha, \mathrm{NV}$ and CIV was observed with HST (Laor 1998a). (3) Mrk 1298 (or PG1126-041): CIV and NV absorption lines are clearly present in the low dispersion, IUE spectrum of this object (Figure 2). The S/N, however, is too low to develop detailed models. To conclude, the NLS1s also support the unified X/UV absorber models.

This now brings me to the last object in the Table, PG1404+266.

\section{1 $P G 1404+266$}

ASCA, ROSAT and HST observations of PG1404+266 are discussed in Ulrich et al. 1999. The X-ray spectrum of the object shows the signature of a $\sim 1 \mathrm{keV}$ absorption feature which may be interpreted as a NeVII-NeX warm absorber (note that this would be different from the OVII/OVIII warm absorbers generally observed with ROSAT). PG $1404+226$ also has an unusually strong Fe $\mathrm{K} \alpha$ emission line. The HST spectrum shows the presence of the CIII* $\lambda 1175.7$ emission line and associated absorption lines of Ly $\alpha, \mathrm{NV}$ and CIV. Ulrich et 


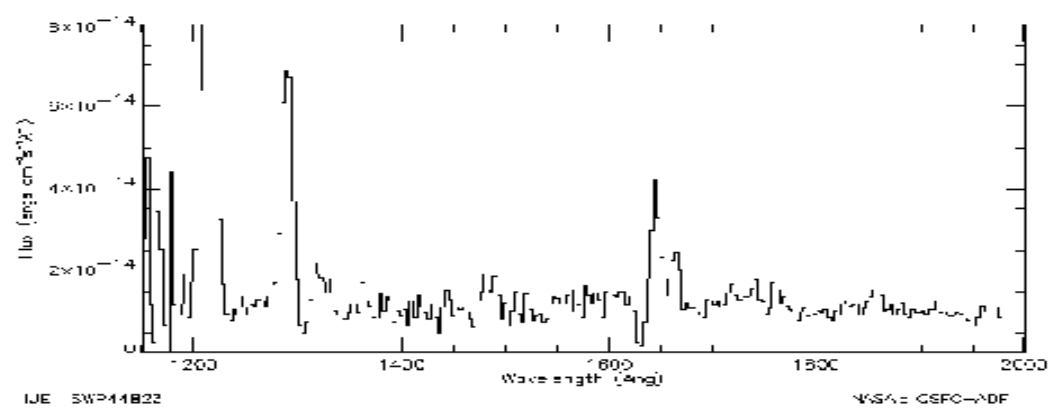

Fig. 2. IUE SWP Spectrum of PG1126-041 showing associated CIV and NV absorption lines (near $1640 \AA$ and $1300 \AA$ respectively).

al. found that, while the strength of the Ly $\alpha$ and CIV lines was generally consistent with that expected from the warm absorber, the strength of NV was not: the NV absorption line was stronger than expected.

This is reminiscent of the metallicity determinations in quasars (see review by Hamann \& Ferland 1999). As shown by Hamann \& Ferland, nitrogen serves as an metallicity indicator. Moreover, nitrogen is preferentially enhanced when metallicities are high, $\mathrm{N} / \mathrm{H} \propto \mathrm{Z}^{2}$. The observations of PG1404+226 thus suggest that nitrogen is enhanced and so the metallicities are super solar in this object and likely to be in NLS1s in general. We are investigating whether this suggestion is correct by performing a detailed metallicity analysis (Mathur \& Komossa 2000).

\section{$3 \quad$ Are NLS1s Active Galaxies in the making?}

In addition to the case of PG 1404+226 discussed above, there are other lines of evidence suggestive of high metallicities in NLS1s. Wills et al. (1999) found that the strength of the NV $\lambda 1240$ emission line was systematically larger while the strength of CIV $\lambda 1549$ was systematically smaller in AGN with narrow emission lines (see also B. Wills, these proceedings). The strength of the fluorescent Fe-K alpha line in some NLS1s is also indicative of a super-solar abundance (A. Fabian, these proceedings). Thus the observations of emission as well as absorption lines in NLS1s imply super-solar gas phase metallicities.

Such metal enrichment is possible when the initial mass function of star formation is flat, favorable for high mass star formation, and the evolution is fast. Such a star formation scenario is likely to be present in deep potential wells 
like galactic nuclei and protogalactic clumps (HF99). Moreover, high metallicities are achieved while consuming less gas (HF99). The NLS1s may then represent that early phase in galactic evolution when rapid star formation is taking place in the nucleus.

Note also that NLS1s have relatively smaller BH masses. As per the well known correlation of Magorrian et al. (1998), smaller mass BHs reside in galaxies with smaller spheroids. Since NLS1s have relatively smaller mass BHs compared to normal Seyferts, the spheroids of their host galaxies might be smaller (see also Laor 1998b). Indeed, in the compilation of Wandel (1999), the NLS1 galaxy NGC4051 has the smallest black hole to bulge mass ratio. An accreting $\mathrm{BH}$ would also grow in mass with time [the Salpeter time scale of growth is determined by $\mathrm{t}_{s}=3 \times 10^{7}\left(L_{E d d} / L_{B}\right) \eta_{0.1} \mathrm{yr}$. where $\eta_{0.1}$ is the radiative efficiency in units of 0.1 (see Fabian 1999)]. Since NLS1s accrete at close to the Eddington limit, their BHs would grow faster. So, smaller BHs in NLS1s are likely to be younger as well.

These arguments support my proposal that NLS1s might be Active Galaxies in early phases of their evolution.

\section{Similarity between NLS1s and the high redshift quasars.}

Hamann \& Ferland (1993) found high metallicities in high redshift quasars $\left(\mathrm{Z}_{\sim} \mathrm{Z}_{\odot}\right.$ at $\left.\mathrm{z} \sim 4\right)$. Similarly we find that NLS1s may also have large metallicities.

Similarities between the observed properties of low ionization Broad Absorption Line Quasars (BALQSOs) and NLS1s have been reported in the literature (e.g. Lawrence et al. 1997, Leighly et al. 1997). Both these classes show strong Fe II $\lambda 4570$ and AlIII $\lambda 1857$ and weak CIV $\lambda 1549$ and [OIII]5007 emission lines. Their continua are red in the optical and strong in the IR. Evidence of relativistic outflow is also reported in three NLS1s (Leighly et al. 1997). If these two classes are indeed related (see also Brandt, these proceedings), then NLS1s, at least those with some evidence of outflow, might be low redshift, low luminosity cousins of BALQSOs. BALQSOs are tentatively identified with a phase in quasar evolution when the matter around the nuclear $\mathrm{BH}$ is being blown away, and a quasar emerges (see, e.g. Fabian 1999). NLS1s may then represent a similar early evolutionary phase at low redshift.

Optical spectra of a sample of $\mathrm{z}_{\sim}^{>4}$ quasars revealed that their emission lines

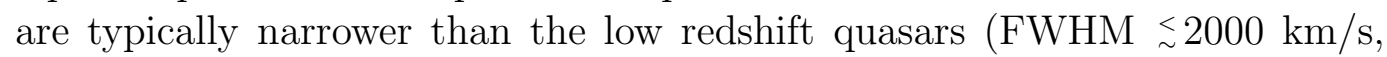
Shields \& Hamann 1997b). The normal explanation of this observation is that these are type 2 quasars, where the broad emission lines are obscured from our line of sight. Alternatively, these high z quasars might be true "narrow" 
broad line objects (see Mathur 2000 for a discussion of selection effects).

There is another interesting connection with high redshift. As discussed above, NLS1s have strong Fe II emission lines. Quasars Q0014+813 and Q0636+680 at redshifts $\mathrm{z}=3.398$ and $\mathrm{z}=3.195$ respectively, were observed to have very strong Fe II emission (Elston, Thompson, \& Hill 1994). Are they also highly accreting objects at an early evolutionary phase? Note also the narrow UV emission lines (FWHM $\underset{\sim}{\sim} 150 \mathrm{~km} \mathrm{~s}^{-1}$ ) in the ultra strong UV Fe II emitter Q2226-3905 (Graham, Clowes, \& Campusano, 1996).

All these similarities point towards NLS1s being low redshift, low luminosity analogues of high redshift quasars.

\section{Do NLS1s reside in rejuvenated galaxies?}

I have argued that NLS1s may represent an early phase in AGN evolution. Whether they reside in young galaxies is a separate question and a step further. That young galaxies are gas rich is helpful; they would have the large reservoir of gas necessary to sustain the close to Eddington-rate accretion in NLS1s. But do we have any evidence that they indeed reside in young galaxies? There is no published systematic study of the properties of the host galaxies of NLS1s. However, some of the NLS1s are originally from the Zwicky (e.g. I Zw 1) and Markarian (e.g. Mrk 766) samples of galaxies implying that they are blue. While the blue color might be due to big blue bumps in the active nuclei, as in normal Seyfert galaxies, NLS1s have weak blue bumps and so the blue colors might be a result of actively star forming galaxies. Some NLS1s (e.g. IRAS 13349+2438), are infrared luminous, and star forming. Using the galaxy catalogs RC3 (de Vaucouleurs et al. 1991) and UGC (Nilson 1973), we looked into the morphology of a small sample of NLS1s listed in Table 1 and found information on seven of them. Three were found to be compact (I Zw 1, Mrk 507, and Mrk 1298), two showed signatures of an inner ring (NGC 4051 and Ark 564), and three have nuclear bars (NGC 4051, Mrk 766 and Ark 564). These are signatures of recent activity (though not necessarily), quite likely due to galaxy-galaxy interactions or mergers. In this scenario the galaxies are newly formed, or rejuvenated.

That NLS1s reside in young galaxies is also consistent with the hypothesis that the formation and evolution of galaxies and their active nuclei is intimately related (Rees 1997, Fabian 1999, Granato et al. 1999, Haehnelt \& Kauffmann 1999). In this scenario, the process of formation of a massive $\mathrm{BH}$ and the active nucleus is the very process of galaxy formation. The active nucleus and the galaxy evolve together, with the $\mathrm{BH}$ accreting matter and the galaxy making stars. At one stage the winds from the active nucleus blow away the 
matter surrounding it and a quasar emerges. This is not only the end of the active evolution of the quasar, but of the galaxy as well, as it is evacuated of its interstellar medium. The quasar then shines as long as there is fuel in the accretion disk (Fabian 1999). In this scenario, high redshift quasars represent an early stage of galaxy evolution, BALQSOs at $\mathrm{z} \approx 2$ represent the stage when the gas is being blown away, and $\mathrm{z} \approx 1$ quasars would be the passively evolving population. Massive ellipticals found today might be the dead remnants of what were once quasars.

The quasar phenomenon may thus be a result of galaxy formation due to primordial density fluctuations. At low redshift, when new galaxies are formed due to interactions or mergers, similar evolution may take place. As argued above, the NLS1s may represent a crucial early phase. (In our scenario, the accretion rate $\dot{\mathrm{m}}=\dot{\mathrm{M}} / \dot{\mathrm{M}}_{E d d}$ is large in the early stages of evolution and reduces later on. This is opposite to the proposal by Wandel (1999) in which $\dot{\mathrm{m}}$ increases with time.)

In fact, there might be some NLS1s with a starburst component. Soft X-ray spectra of NLS1 are steep and often variable. However, Leighly et al. (1996) and Page et al. (1999) report that while the power-law component in the NLS1 Mrk 766 varied, the thermal black-body component did not. This component might well be due to a nuclear starburst. Note also the strong CO emission in the prototype NLS1 I Zw 1 (Barvainis, Alloin \& Antonucci 1989). Schinnerer, Eckart \& Tacconi (1998) mapped I Zw 1 in CO and found a circumnuclear ring of diameter $1.8 \mathrm{kpc}$. The authors found strong evidence for a nuclear starburst. There is also a companion to I Zw 1, supporting an interpretation of the starburst being due to an interaction. Similarly, AGN activity is known to exist in starburst galaxies (see Heckman 1999 for a review). The poster by Dennefeld et al. reports observations of narrow optical emission lines in a sample of IR selected starburst galaxies. See Mathur (2000) for the details of the connection between NLS1s and the evolution of galaxies and active galaxies.

\section{On strong Fe II emission in NLS1s.}

There is a general consensus that large accretion rate, $\dot{\mathrm{m}}$, is a possible driver of many of the observed properties of NLS1s. Strength of Fe II emission may also be linked to the large accretion rate. In a model by Kwan et al. (1995), Fe II line emission is produced in an accretion disk. The accretion disks with larger accretion rate may simply have more mass to produce stronger Fe II. Thus I would like to argue in favor of collisional ionization as the origin of the Fe II emission in NLS1s and AGNs in general (see also the article by S. Collin in these proceedings). Remember also the CIII $^{*} \lambda 1174.7$ observed in PG 
1404+266. This line is also seen in the HST spectrum of another NLS1, IZw 1 (Laor et al. 1997). Laor et al. preferred the explanation that it is produced by resonance scattering of continuum photons. However, in this scenario the velocity gradient in each emitting cloud and the total covering factor would be exceptionally large. The presence of the CIII* $\lambda 1174.7$ line, together with strong Fe II emission, suggests a collisional origin.

\section{Conclusions}

That the AGN phenomenon was so much stronger at $\mathrm{z} \sim 2-3$ than today has long elicited the suspicion that there is a connection between the youth of a galaxy and the likelihood that an AGN forms inside it. The question then naturally arises, "what are the local counterparts to the young galaxies in the early universe in which local AGN may live?" (Krolik 1999). A standard answer to this question is "Starburst galaxies". Heckman (1999) has argued that starburst galaxies are the low redshift analogues of Lyman break galaxies at high redshift. Similarly, we ask, what are the low redshift analogues of high redshift $\left(\mathrm{z}_{\sim}>4\right)$ quasars? I propose that they might be NLS1s.

It is my pleasure to thank Th. Boller and the organizing committee for inviting me to this wonderfully stimulating workshop. I thank the Wilhelm and Else Heraeus foundation for travel support and the delightful stay at the Physikzentrum, Bad Honnef. This work is supported in part through NASA grant NAG 5-3249 (LTSA). The figures were created using the archives at the Space Telescope Science Institute, operated by the Association of Universities for Research in Astronomy, Inc., from NASA contract NAS5-26555.

\section{References}

[1] Brandt, W. N., Mathur, S., Reynolds, C. S., Elvis, M. 1997 MNRAS 292, 407

[2] Barvainis, R., Alloin, D., \& Antonucci, R. 1989 ApJL, 337, L69

[3] Crenshaw, M. 1997 in ASP Conference Series Vol. 128 "Mass Ejection from AGN", Ed.: N. Arav, I. Shlosman, \& R. J. Weymann [ASP: San Francisco]

[4] Elston, R., Thompson, K. \& Hill, G. 1994, Nature, 367, 250

[5] Fabian, A.C. 1999, MNRAS, 308, L39

[6] Graham, M., Clowes, R. \& Campusano, L. 1996, MNRAS, 279, 1349

[7] Granato, G.L., Silva, L., Monaco, P., Panuzzo, P., Salucci, P., DeZotti, G., \& Danese, L. 2000, MNRAS, submitted (astro-ph/9911304)

[8] Haehnelt, M. \& Kauffmann, G. 1999, astro-ph/9911514 
[9] Hamann, F. \& Ferland, G. 1993, ApJ, 418, 11

[10] Hamann, F. \& Ferland, G. 1999, ARAA (astro-ph/9904223)

[11] Heckman, T. 1999 (astro-ph/9912029)

[12] Krolik, J. 1999 "Active Galactic Nuclei" [Princeton]

[13] Kwan, J. 1984, ApJ, 283, 70

[14] Kwan, J., Cheng, F., Fng, L., \& Ge, J. 1995, ApJ, 440, 628Wandel, A., 1999, ApJL, 519, 39

[15] Laor. A. 1997 in "Emission Lines in Active Galaxies: New methods and Techniques", Ed: B. M. Peterson, F.-Z. Cheng, and A. S. Wilson. [ASP Conference Series 113]

[16] Laor. A. 1998a, in "Quasars and Cosmology", Ed: G. Ferland, J. Baldwin [ASP Conference Series 162]

[17] Laor. A. 1998b, ApJL, 505, 83

[18] Lawrence, A.; Elvis, M.; Wilkes, B. J.; McHardy, I.; Brandt, N. 1997, MNRAS, 285, 879

[19] Leighly, K., Mushotzky, R., Yaqoob, T., Kunieda, H., Edelson, R. 1996 ApJ 469, 147

[20] Leighly, K., Mushotzky, R., Nandra, K., \& Forster, K. 1997, ApJ, 489, 25

[21] Magorrian, J. et al. 1998, AJ, 115, 2285

[22] Mathur, S. 1994 ApJL, 431, L75

[23] Mathur, S., Wilkes, B. J., Elvis, M. S., \& Fiore, F. 1994 ApJ, 434, 493

[24] Mathur, S., Elvis, M. S. \& Wilkes, B. J. 1995, ApJ, 452, 230

[25] Mathur, S., Wilkes, B. J., \& Aldcroft, T. 1997, ApJ, 478, 182

[26] Mathur, S. 1997 in ASP Conference Series Vol. 128 "Mass Ejection from AGN", Ed.: N. Arav, I. Shlosman, \& R. J. Weymann [ASP: San Francisco]

[27] Mathur, S., Wilkes, B. J., \& Elvis, M. S. 1998, ApJL, 503, 23

[28] Mathur, S., Elvis, M. S., \& Wilkes, B. J. 1999, ApJ, 519, 605

[29] Mathur, S., 2000, MNRAS Letters, in press.

[30] Mathur, S., \& Komossa, S. 2000, in preparation

[31] Page, M. J., Carrera, F. J., Mittaz, J. P. D., \& Mason, K. O. 1999, MNRAS, 305, 775

[32] Rees, M. 1997, in "Black Holes and Relativity", ed. R. Wald.

[33] Shields, J., \& Hamann, F. 1997a ApJ, 481, 752

[34] Shields, J., \& Hamann, F. 1997b in 1st Guillermo Haro Conference on Astrophysics: Starburst Activity in Galaxies, Puebla, Mexico, p. 221

[35] Schinnerer, E., Eckart, A., \& Tacconi, L. 1998, ApJ, 500, 147

[36] Ulrich, M.-H., Comastri, A., Komossa, S., \& Crane, P. 1999, A\&A, in press (astro-ph/9907150)

[37] Wandel, A., 1999, ApJL, 519, 39

[38] Wills, B., Brotherton, M.S., Laor, A., Wills, D., Wilkes, B.J., Ferland, G., \& Shang, Z. 1999 in "Quasars and Cosmology", Ed. Gary Ferland and Jack Baldwin. [ASP Conference Series volume 162] 